\title{
METODOLOGIA DE ANÁLISE SOCIOLÓGICA DISCURSIVO-IMAGÉTICA: POSSIBILIDADES AOS ESTUDOS ORGANIZACIONAIS
}

\author{
Discursive-imagetic Sociological Analysis Methodology: \\ possibilities for organizational studies
}

\author{
Christiane Kleinübing Godoi* \\ Antônio Giovanni Figliuolo Uchôa**
}

\section{RESUMO}

Existe no campo das ciências sociais um espectro de discussões sobre imagens que data de mais de quatro décadas. Não emergiu ainda um método de interpretação específico que se ocupe diretamente do nível visual - em seus diferentes objetos - sem transcrever, previamente, sob a forma descritiva para, posteriormente, realizar algum tipo de análise textual ou discursiva sobre a transcrição. 0 objetivo deste estudo reside em sistematizar uma metodologia integradora de diferentes níveis de aproximação da imagem por meio das seguintes etapas: a) delimitação da cultura visual contemporânea; b) narrativa da historicidade das práticas de análise de imagens originárias em outras ciências visuais; c) estabelecimento de dialogicidade entre a metodologia da Análise Sociológica do Discurso (ASD) com traslado e reconceituação desta perspectiva para o campo da Análise Sociológica da Imagem (ASI); d) construção de uma metodologia híbrida integrando as duas metodologias anteriores, ou seja, Análise Sociológica Discursivo-Imagética.

Palavras-chave: Análise Qualitativa de Material Visual. Análise de Imagens. Metodologias Visuais.

\begin{abstract}
In the social and human sciences, theoretical discussions about images date back over four decades. No specific method of interpretation has yet emerged that deals directly with the visual level, in its different objects, without previously transcribing it, in descriptive form, so that it can then be analyzed textually or discursively. The objective of this study is to systematize a contextualized and integrative methodology of different levels of image approximation through the following steps: a) delimitation of the sociocultural context in which the discussion about image analysis emerges; b) narrative of the historicity of the practices of analysis of images originating in other sciences; c) establishment of dialogue between the methodology of the Sociological Discourse Analysis (SDA) with evaluation of possible new concept transfer from this perspective to the field of Sociological Image Analysis; d) construction of a hybrid methodology which integrates both methodologies as a Sociological Discursive-Imagetic Analysis.
\end{abstract}

Keywords: Qualitative Analysis of Visual Material. Image Analysis. Visual Methodologies.

\footnotetext{
* Pós-Doutora em Sociologia pela Universidad Complutense de Madrid (Espanha). Doutora em Engenharia de Produção pela Universidade Federal de Santa Catarina (UFSC). Professora do Programa de Pós-graduação da Universidade do Vale do Itajaí (Univali) - Itajaí (SC), Brasil. E-mail: christiane.godoi@yahoo.com.br. ORCID: 0000-0002-0627-4833

** Doutor em Administração pela Universidade do Vale do Itajaí (Univali). Professor Adjunto da Faculdade de Estudos Sociais da Universidade Federal do Amazonas (UFAM) - Manaus (AM), Brasil. E-mail: antoniouchoa@ufam.edu.br. ORCID: 0000-0002-0451-889X
} 


\section{INTRODUÇÃO}

C onsidera-se surpreendente que a pesquisa qualitativa se limite, até o momento, apenas a incorporar a multiplicidade de materiais visuais produzidos em diferentes contextos, com diferentes objetivos específicos - fotografias, desenhos, pinturas, filmes, apresentações teatrais, publicações comerciais, programas televisivos, videoclipes, revistas femininas, jornais, ilustrações variadas, contos, plataformas virtuais, esculturas. Para exemplificar importantes trabalhos desta tipologia - aplicados a único objeto imagético - cabe mencionar trabalhos históricos seminais: a) Harper (2000), ao tecer ampla retrospectiva da técnica de foto-elicitação mostrando fotografias aos sujeitos da pesquisa; b) o artigo marcante também de Harper (2003), realizando uma retrospectiva da etnografia visual e seus arquivos fotográficos; e c) o conhecido estudo de Bateson e Mead (2012), igualmente sobre fotografias.

No cenário dos Estudos Organizacionais, faz-se necessário referenciar o estudo inaugural de Caldas e Tonelli (2002) - o primeiro no país a trabalhar com desenhos na compreensão de fenômenos organizacionais. Entretanto, permanecemos até o momento, em nossa área, neste mesmo estágio de 20 anos atrás, prescindindo-nos da dedicação mais atenta em desenvolver metodologias mais amplas de tratamento deste tipo de material cada vez mais emergente na sociedade. Esta compreensão de paralisia do desenvolvimento de técnicas ou práticas "uni-objetais" (destinadas somente a um único objeto imagético, como desenhos ou fotografias, por exemplo), por mais completas e fundamentadas que possam ser (ver, por ex: ALCADIPANI; TONELLI, 2014; CUNLIFF; ALCADIPANI, 2016), não faz parte do escopo teórico-metodológico desta proposta. Entendemos Metodologia de Análise Sociológica Discursivo-Imagética aqui desenvolvida, não como simples ato de analisar discursos e imagens, mas como todo o percurso, o caminho do pensamento para tornar 0 pesquisador capaz de utilizar um objeto imagético (lendo-o "como se fosse discurso" - esta questão ficará esclarecida no último e principal capítulo), com a finalidade de produzir conhecimento no campo dos Estudos Organizacionais. Não se trata de "como analisar", de uma simples "técnica de análise de dados", mas da criação de um espaço metodológico no qual pesquisadores de diferentes epistemes, que trabalham com diferentes objetos imagéticos e perspectivas de análise possam debater e circular.

Acerca do contexto da cultura material-visual contemporânea, parodiando a conhecida "guinada linguística" ocorrida no interior da Filosofia da Linguagem, Mirzoeff (2003) e Serrano (2008) utilizam a expressão "guinada visual" a fim de caracterizar a onipresença do espectro audiovisual em nossa sociedade. Mesmo ponto de partida e raciocínio é empregado por Chalfen (2011), Meyer et al. (2013), Wagner (2011b) - na medida em que caracterizam a realidade imagética como traspassada pelo modo visual do discurso e da construção do significado.

Ainda que atrelada a contemporaneidade, a presença de imagens como objeto de estudo no interior de determinadas ciências sociais data de décadas, e o que se assiste hoje é um elevado crescimento em todas as áreas de interesse de pesquisadores originários de diversas orientações disciplinares, como a antropologia, a sociologia, a história, a psicologia social, a comunicação, e os Estudos Organizacionais em dar conta de debruçar-se sobre objetos imagéticos diversos para explicar e compreender fenômenos sociais, revelando, sim, 
um crescente interesse por métodos de análises visuais (ver, por exemplo: GARCÍA-VERA; MAILLO, 2011; GUASCH, 2006; JACKSON, 2005; MENÉNDEZ; RODRÍGUEZ, 2012; PAUWELS, 2011; REBOLLO, 2002; ROSE, 2012; SERRANO, 2012; WAGNER, 2011a).

Entretanto, até o momento, não surgiu - em nenhuma disciplina do conhecimento - um método de interpretação que se ocupe direta e exclusivamente do nível visual, sem proceder a transcrições antecedentes, como criticam o renomado metodólogo Flick (2004); García-Vera e Maillo (2011). Para fundamentar tal crítica, os artigos por nós encontrados acerca das imagens ou visualidades: a) ou são teorizações filosóficas e sociológicas, como a narrativa geral sobre o crescimento das metodologias visuais, tal como a de Mirzoeff (2002), que tece a ascensão dos estudos sobre imagens desde análises com o "Panopticon de Bentham"; e o estudo de Rieger (2003) nos EUA, que elabora uma retrospectiva do crescimento dos estudos visuais a partir das mudanças sociais; ou b) consistem em simples "técnicas de análise" que se destinam a um único objeto imagético exclusivo, em geral fotografias, tal como o clássico Margolis e Rowe (2011). Com raras exceções como o estudo metodológico empreendido por Meyer et al. (2013), que intenciona organizar a temática, a literatura acerca do tema é fragmentada e não há consenso entre os autores acerca do que sejam tendências, abordagens, métodos, técnicas de análise visual. Na crítica de Flick (2004); García-Vera e Maillo (2011), portanto, o que se tem feito até o momento é, diante da imagem, transcrever sob a forma de relações descritivas para, posteriormente, fazer uma análise textual ou discursiva em cima do texto gerado na transcrição da imagem. Os autores críticos destas práticas são enfáticos em afirmar que as codificações, categorizações e interpretações deveriam ser feitas diretamente sobre o material audiovisual. No entender de renomado metodólogo Flick (2004), é necessário, portanto, que a análise do discurso deva fazer-se diretamente sobre o material visual a fim de evitar, por exemplo, esquecer componentes não verbais contidos neles.

A carência de emprego de metodologias visuais de maior aprofundamento e rigor, envolvendo considerações sobre o entendimento da própria pesquisa empírica, o impacto da imagem sobre a vida social, suas origens ao cruzar as fronteiras disciplinares na construção de diálogo com diferentes pensamentos acadêmicos, também é alvo da crítica de metodólogos como Wagner (2011a), Arroyo e Sábada (2012), e Arroyo (2012). No campo da sociologia aplicada, no qual residem os Estudos Organizacionais, observa-se ainda maior escassez literária sobre a temática, quando muito é restrita à descrição de procedimentos metodológicos nos campos da opinião pública, e de mercado, por exemplo, que tratam de estudos de percepção da imagem. Mesmo que as análises fílmicas e fotográficas tenham advindo para o campo das ciências sociais a partir da antropologia, é de método que nos interessa falar - no centro da investigação em Estudos Organizacionais -, ou seja, de que maneira têm sido utilizados os registros imagéticos na pesquisa em ciências sociais. Noutros termos, passar a considerar a urgência e o potencial latente que tem os materiais visuais ao serem incorporados na análise de fenômenos sócio organizacionais.

Portanto, com a finalidade última de contribuir para a criação de um espaço profundo e permanente de debate, discussão, sistematização de uma Metodologia de Análise Qualitativa de Material Visual no interior dos Estudos Organizacionais, este trabalho teórico-metodológico está subdivido nas seguintes etapas: a) delimitação do contexto sociocultural em que vem emergindo a inserção de técnicas e práticas ligadas à análise de imagens, ou seja, o cerne da cultura material contemporânea; b) narrativa da historicidade das práticas 
e técnicas de produção e de análise de material visual originárias em outros campos do conhecimento, como antropologia visual, história visual e sociologia visual; c) estabelecimento de dialogicidade com o método e os procedimentos da Análise Sociológica do Discurso avaliando as possibilidades de traslado reconceituado desta perspectiva também sócio-hermenêutica e pragmática para o campo da análise visual; d) abertura de possibilidade de construção de uma perspectiva integradora e contextualizada destas diferenças que seja destinada exclusivamente à análise direta de material visual e, por fim, passível de ser utilizada com diferentes objetos imagéticos em si.

\section{CULTURA VISUAL-MATERIAL COMO CONTEXTO DE ANÁLISE DE IMAGENS}

Serrano (2008) - principal representante da Tradição Espanhola de Pesquisa Social Qualitativa no que tange à análise de imagens - historiciza que o termo cultura visual foi usado pela primeira vez por Alpers, em 1983, referindo-se à sociedade holandesa do século XVII (ALPER; VERMEER, 1983). Recentemente, na análise de Serrano (2008) e Rose (2012), o termo foi retomado e retornou a ser utilizado por estudiosos da sociedade contemporânea para explicar a forma como as tecnologias visuais passaram a "desbancar" os textos escritos, além de terem-se convertido em uma das principais formas de comunicação, passaram a constituir um dos marcos fundamentais da sociedade pós-modernas, ou também chamadas, sociedades iconocentradas (ROSE, 2012). Inserir a discussão neste cenário dos estudos da cultura da imagem (WAGNER, 2011b) tem como missão buscar não incorrer em mais uma forma proposta de que o objetivo não é alcançar o que está abaixo da superfície da imagem, alertam Serrano e Zurdo (2012), mas sim ampliá-la, enriquecê-la, dar-Ihe definição e tempo. Entendemos que imagens são - assim como o discurso - práticas culturais (SERRANO; ZURDO, 2012) que carregam os valores daqueles que as criam, manipulam e consomem.

Cultura material (JAKSON 2005; LIEBENBERG, 2009; MIRZOEFF, 2002), mais especificamente, refere-se a objetos que são usados, vivenciados, exibidos e experimentados em determinada cultura. Os seres humanos interagem com a cultura material como uma parte normal de suas vidas cotidianas. Devido a esta interação, cultura material e vida humana são fortemente influenciadas pelo Outro, de tal forma, que estudar essa cultura material pode nos dar pistas importantes sobre a forma como os seres humanos vivenciam o cotidiano por meio de imagens (O'TOOLE; WERE, 2008). A forma como pensamos a cultura material, caminha de mãos dadas com a forma como refletimos sobre a cultura, e molda também as idiossincrasias sob as quais abordamos os estudos visuais da cultura e da vida social (WAGNER, 2011a). Embora alguns teóricos influentes da cultura material tenham enfatizado a objetividade do artefato, Thomas (2009) só concebe reconhecer 0 inverso: a mutabilidade das coisas na reconceituação. Coleções de museus e exposições, filmes e documentários em videocassete e livros ricos em fotografia são exemplos, elencados por Wagner (2011a), de uma vitalidade contínua a esta orientação para a cultura material.

No entender de Serrano e Zurdo (2012), não existe, portanto, um olhar inocente, mas sempre condicionado por pressupostos culturais, ideologias, estereótipos, modas, deformações profissionais, representações inconscientes, experiências e motivações. Cultura material - formada por objetos e imagens - condiciona, por duas vezes, as heurísticas do 
sujeito ator e receptor dessa cultura imagética. Até aqui, poderíamos afirmar que, tal como em análises discursivas, o sujeito do olhar é também um sujeito social. Não existe, por este motivo, a ilusão de uma linguagem de imagens (BANKS; ZEITLYN, 2015) ou componentes de imagem que seguem algum tipo de regra quase gramatical universal, mas ao contrário, tais componentes só fazem sentido em contextos socialmente mais específicos.

A cultura visual-material da contemporaneidade postula que a imagem substitui a realidade, criada de forma espetacular, um simulacro, para ser materialmente consumida Sabemos que o sujeito autorreferencial da pós-modernidade ocularcêntrica (ROSE, 2012) - uma cultura visual - perdeu suas grandes instituições e narrativas referenciais e passou a viver em um mundo de informações tecnologizadas (SERRANO; ZURDO, 2012) criadas para serem consumidas. Esta é, no fundo, a finalidade última das imagens produzidas pela cultura material, que Felstead, Jewson e Walters (2004) consideram como sempre ambígua, com significados múltiplos e incerta. Este sujeito pós-humano não é engajado com o mundo, proclama Rose (2012), por meio de significados interpretados e compartilhados e, sim, por experiências sensoriais e perceptivas de imagens.

Passamos neste momento à análise da historicidade do uso de imagens como ciência no interior das ciências sociais puras.

\section{ORIGENS DA UTILIZAÇÃO DAS TECNOLOGIAS VISUAIS EM CIÊNCIAS SOCIAIS:}

0 OLHAR DA ANTROPOLOGIA, HISTORIA E SOCIOLOGIA VISUAL

O olhar etnográfico sobre o campo traduziu-se sobre experiências com câmera e filme que remontam ao século XIX, especificamente a uma das primeiras fases antropológicas de familiarização do investigador com seus informantes (REBOLLO, 2002). Mead (2003) já alertava que a imagem ainda permaneceria em seu caráter subalterno por muito tempo, de maneira ilustrativa ou de divulgação, em uma ciência na qual a antropologia era descrita por práticas tradicionais, e envolvendo a confiança nas palavras, narrada e escrita.

No campo da Sociologia Visual - mais próximo dos Estudos Organizacionais (Sociologia Aplicada), registros de imigrantes europeus foram capturados no início do século XX pelo sociólogo Lewis Hine, documentando a chegada destes à Ellis Island, Nova York (EUA) (COLLIER; COLLIER, 1986). Outro estudo como o de Jackson (1977), nas penitenciárias do Arkansas (EUA), procurou detalhar a vida cotidiana na prisão. Neste esforço para capturar e analisar as realidades das práticas sociais, tecnologias fotográficas e de vídeo provaram inestimável fornecimento de registros dos detalhes das ações em quatro categorias, considerando: (1) as fotografias geradas pelo pesquisador como dados gerados para caracterizar o ambiente social local, ao complementar a pesquisa observacional (BANKS, 2012); (2) vídeo gerado pelo pesquisador, no apoio à investigação sobre a ordem das ações em lugares públicos (BANKS; ZEITLYN, 2015); (3) áudio e vídeo gerado pelo pesquisador, como registro de gravações sobre a organização prática do trabalho de descobertas científicas (GARFINKEL; LYNCH; LIVINGSTON, 1981); (4) Registro de áudio-vídeo gerado pelo pesquisador dos usos originais de imagens e vídeo, neste caso registrando como profissionais usam imagens e vídeo como parte do seu trabalho, a exemplo de cirurgiões ensinando a usar vídeos em cirurgias (MONDADA, 2008). 
Nesse sentido, práticas denominadas como a refotografia e foto-elicitação constituem práticas visuais inovadoras, geralmente associadas não somente ao campo da sociologia visual. A técnica de foto-elicitação, já em meados 1950, auxiliava na produção de estudos como uma poderosa ferramenta que utilizava a dimensão visual para coletar informações junto aos entrevistados. Seu princípio está associado ao experimento de Collier Jr. (1957) denominado inicialmente como foto-entrevista, expondo fotografias aos entrevistados durante as entrevistas, para um melhor entendimento dos fenômenos sociais e culturais (MEYER et al., 2013). A compreensão do significado da imagem é explorada pelo pesquisador e o entrevistado durante a conversação - uma interpretação participante com descrição verbal - é estimulado a intercambiar um diálogo guiado por imagens, dando maior voz ao entrevistado para com suas próprias interpretações (HUGHES, 2012; LAPENTA, 2011; RIEGER, 2003).

O termo "participativo" (MITCHELL; LANGE, 2012), ou "participante" na Sociologia Visual concebe inicialmente as formas com que membros de uma comunidade estão envolvidos com a criação da narrativa de vídeo, incorporam o próprio texto como um catalisador ou um gatilho nas discussões pós-exibição. Descrito por Shaw (2012) com um processo lento e complexo, Mitchell e Lange (2011) minimizam a crítica ao pontuar sobre o vídeo participativo como um processo que favorece a possibilidade de aprofundamento sobre as mudanças sociais da comunidade, comparativamente a outras perspectivas longitudinais envolvendo vários anos de observação. Trabalhar com a produção de vídeo como um processo de grupo, permite aos participantes acesso a um tipo de conhecimento construído socialmente que é particularmente significativo, objetivando a temas que, em geral, constituem um tabu - não falado - criando um forte sentido de resposta coletiva que inclui todos os participantes, concluem Mitchell e Lange (2011). Um violento processo de estruturação, registrado de forma cinematográfica, atribuiu à linguagem visual o poder de discurso social e cultural, ponto este incrementado na escrita de Meneses (2003), ao abordar a sociologia visual e os problemas e estudos visuais associados ao poder.

No interior destas tipologias de práticas distintas, é importante ressaltar que Schwartz (1989) já demonstrava uma preocupação teórica sobre as expectativas não somente sobre o uso das imagens em pesquisa qualitativas sobre distintos pontos de vista ou interpretação - pesquisador e sua audiência -, ao tratar essas imagens e seu entendimento, em um contexto social e interativo a prover significado. Imagens podem, portanto, intuir e manifestar, ou aflorar emoções, recordações, e motivar a locução na investigação de fenômenos sociais.

Cabe ressaltar que, na verdade, recorremos às origens seculares da história, antropologia e sociologia visual - apenas como busca de sustentáculo da construção - em uma ciência aplicada - de uma metodologia que não fosse desgarrada de suas ciências mestres. Porém, nas obras acerca da historicidade, como pode aqui observar o leitor, encontramos apenas estudos seminais e, no mais, práticas tecnicistas, ou seja, uso de objetos imagéticos isolados como forma de produção do conhecimento, porém, sem destaque - na historicidade - para metodologias propriamente ditas de análise visual.

Os subcapítulos subsequentes tratam da construção propriamente dita da abordagem metodológica pretendida neste ensaio. 


\section{CONSTRUINDO UMA METODOLOGIA INTEGRADORA DE ANÁLISE DE IMAGENS: DIFERENTES NIVEIS DE APROXIMAÇÃO E CONTEXTUALIZADA NO CAMPO DA PRODUÇÃO E DA RECEPÇÃO}

\section{AS QUATRO DIMENSÕES DE INTEGRAÇÃO EM ANÁLISE DE IMAGENS}

Após o percurso aqui estabelecido acerca da necessidade de construção no campo dos Estudos Organizacionais de uma metodologia específica e integradora (principalmente que sirva para qualquer tipo objeto imagético) para nortear a produção e a análise de imagens, o que se pretende com este capítulo reside na, já mencionada, construção inicial de uma Metodologia Discursivo-Imagética.

Entendemos por perspectiva metodológica integradora aquela capaz de reunir e viabilizar as seguintes dimensões aqui construídas:

- Dimensão 1 - Diferentes níveis de aproximação da imagem do analista, o que é definido a partir de diferentes correntes, orientações metodológico-epistêmicas e (coluna central da Figura 1);

- Dimensão 2 - a garantia de que tais múltiplas possibilidades metodológico-epistêmicas do investigador, sejam destinadas exclusivamente para a análise direta - sem transcrição a priori - de material visual (observa-se que todos os níveis de aproximação recaem diretamente sobre a coluna central da Figura 1 - a imagem em si, portanto, de forma direta);

- Dimensão 3 - a escolha de diferentes objetos imagéticos em si; e, por fim, a

- Dimensão 4 - capacidade de assegurar que a porta de entrada do pesquisador nesta seara não ignore o contexto social de produção e de recepção da imagem tratada (colunas dois e quatro da Figura 1).

Este último aspecto diz respeito ao fato de a abordagem metodológica ser contextualizada em termos de compreender o contexto de produção e o contexto de recepção da imagem - o que será esclarecido mais adiante.

Acerca das atreladas dimensões 1 e 2 - postura epistemológica e nível de aproximação da imagem utilizada pelo analista -, primeiramente cabe mencionar os principais Níveis de Aproximação do Material Visual - para os quais Rose (2012) e Serrano (2008) estabelecem a seguinte classificação em sequência de profundidade do foco (coluna 1 da Figura1):

a) análise e interpretação composicional (utilizada, na maioria das vezes pelo campo da história da arte para compreender texturas, cores etc.);

b) análise do conteúdo;

c) semiologia/semiótica;

d) psicanálise;

e) análise foucaultiana do discurso; análise crítica anglo-saxã do discurso. 


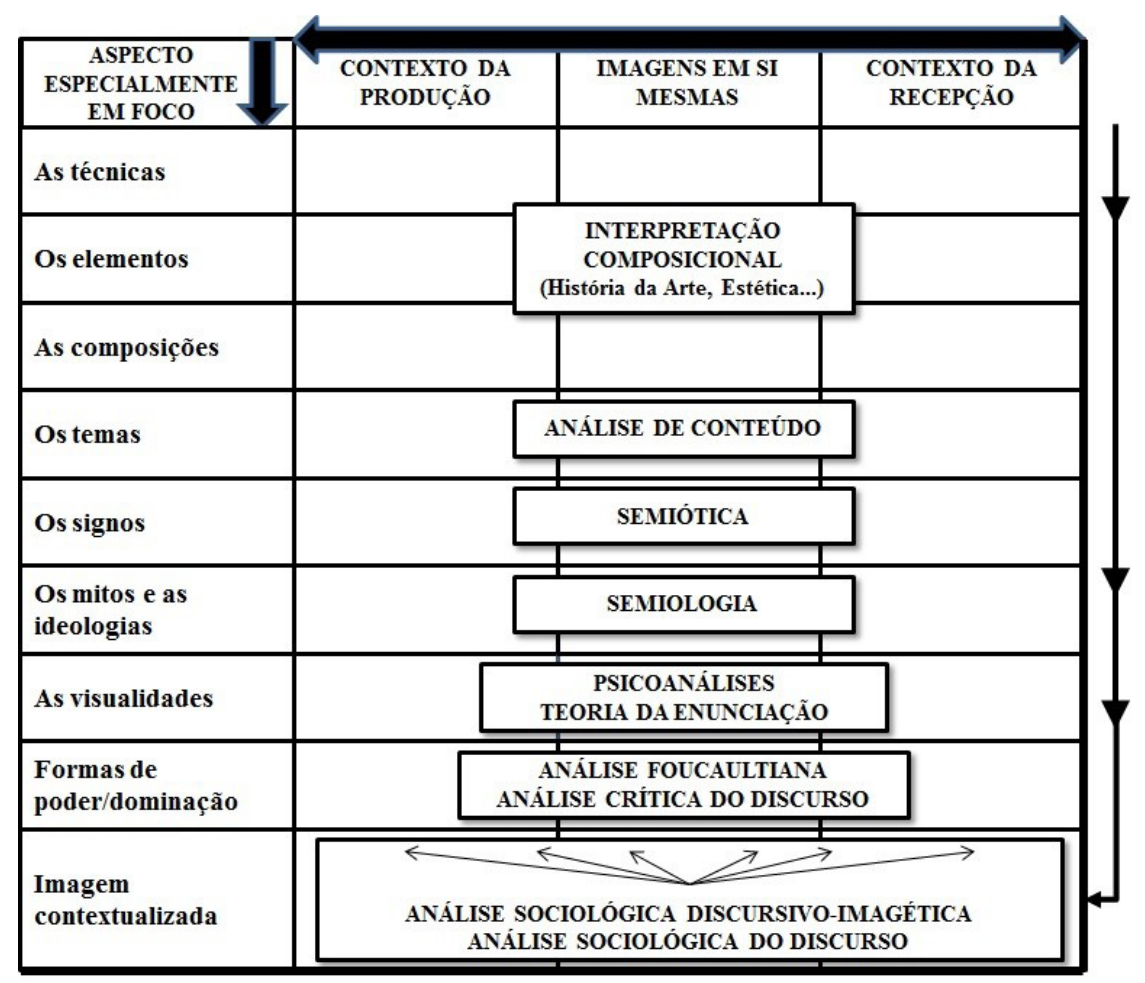

Figura 1 - Níveis de aproximação da imagem

Fonte: Construída pelos autores a partir das obras de Serrano (2008) e Rose (2012).

Com base nestes níveis de aproximação e de profundidade é que se pretende aqui dar continuidade ao trabalho das autoras, incorporando sobre o foco da imagem contextualizada, a possibilidade de interface e traslado - para o cenário visual - da já conhecida nos Estudos Organizacionais brasileiros Análise Sociológica do Discurso (ASD) (CONDE, 2009; GODOI; COELHO; SERRANO, 2014; GODOI, 2010; IBÁÑEZ, 2003; SERRANO 2008) metodologia esta que também trabalha com a noção de discurso contextualizado no espaço social - referindo-se a uma análise sócio-hermenêutica e pragmática.

A Dimensão 3 da metodologia integradora corresponde aos diferentes objetos imagéticos, também chamados de tecnologias imagéticas usados na produção da imagem. Cabe um aprofundamento mais amplo - em virtude de ser esta uma metodologia contextualizada - acerca da Dimensão 4. Com base no trabalho de Rose (2012), Serrano (2008) advoga as diferentes formas de analisar o material visual, a partir de três lugares ou contextos sociais simultâneos:

a) O Contexto de Produção da Imagem (diríamos, de que lugar social provém a imagem construída);

b) O lugar da Imagem em Si mesma e, por último,

c) O Contexto da Recepção da Imagem (ou seja, quem é o sujeito social de onde provém o olhar sobre a imagem, ou ainda, a própria audiência). 
Ao delimitar estes três contextos, Serrano (2008) considera que as práticas de análise de imagens elegidas têm implicações inclusive na seleção de materiais (imagem itself - objeto - tipo de material produzido). É preciso interpretar imagens (quer no contexto da produção quer no da audiência) considerando sentido social, ideológico, cultural, códigos e a capacidade de significar dos documentos texto-visuais ou discursivo-visuais.

O lugar da Produção da Imagem envolve a intencionalidade, o para que (SERRANO, 2008), o processo de criação e difusão. A autora explica que este contexto envolve relações econômicas, sociais, políticas, instituições e práticas que circunscrevem a imagem, de forma que a compreensão deste meio auxilia no entendimento posterior do impacto e efeito a ser produzido pela imagem. O lugar da Imagem em Si Mesma, do objeto imagético não é de fácil compreensão pelo pesquisador iniciante na temática. Desde o interior da sua abordagem epistemológica há que se considerar a materialidade, o significado e a organização espacial de inúmeros elementos que compõem a imagem:

i. Signos e símbolos que a compõe;

ii. O lugar onde se situam os tipos de planos;

iii. O ritmo das sequências;

iv. O que chama atenção nos objetos, formas, cores, recursos utilizados (icônicos, linguísticos, sonoros);

v. Os personagens, relações que exibem e as que não explicitam - em síntese - as cosmovisões (SERRANO, 2008) implicadas.

O Contexto de Recepção da Imagem é o lugar, a identidade, a posição, a expectativa e os interesses dos diferentes tipos de audiências, incluindo o analista. Em síntese, o contexto sócio histórico concreto da recepção (SERRANO, 2008; ROSE, 2012).

Ao revisitar o diálogo estabelecido por Rose (2012) e Serrano (2008) - aqui esclarecido e sintetizado sob a forma de quatro dimensões integradoras e contextualizadas observamos que no interior da Figura 1 a proposta de análise de imagens a partir da ASD foi apenas sobreposta sobre a imagem em si por Serrano (2008), permanecendo desprovida de sistematização de modalidade tecnológica, ou seja, como fazer. Estabelecemos, portanto, um escalonamento hierárquico necessário entre as dimensões, a fim de fazer emergir o lócus da perspectiva aqui pretendida (Figura 2) - Análise Sociológica Discursivo-Imagética (cabe lembrar, "ler imagens como discursos").

O prosseguimento da abordagem integradora, aqui iniciada, prolonga-se no campo dos Estudos Organizacionais por múltiplos caminhos, mas essencialmente por descrever e destrinchar as visualidades (como a visão é construída de várias formas, inclusive inconscientes), tendo em mente que um analista interessado no nível mais superficial das técnicas composicionais incorre em equívoco e incoerência epistemológica, ao atravessar, num "vai e vêm" aleatório, todos os níveis intermediários e sair, a fazer, por exemplo, análise discursiva pura. O que a proposta traz à tona é não apenas a necessidade de compreensão do todo, mas - resguardadas as devidas intencionalidades (visualidades) concretas do pesquisador e suas influências metodológico-epistêmicas -, aquilo que tange a prática de interpretação 
de imagens. Trata-se de criar um cenário sobre o qual o conjunto de abordagens e práticas sobre análise de imagem possa transitar e dialogar.

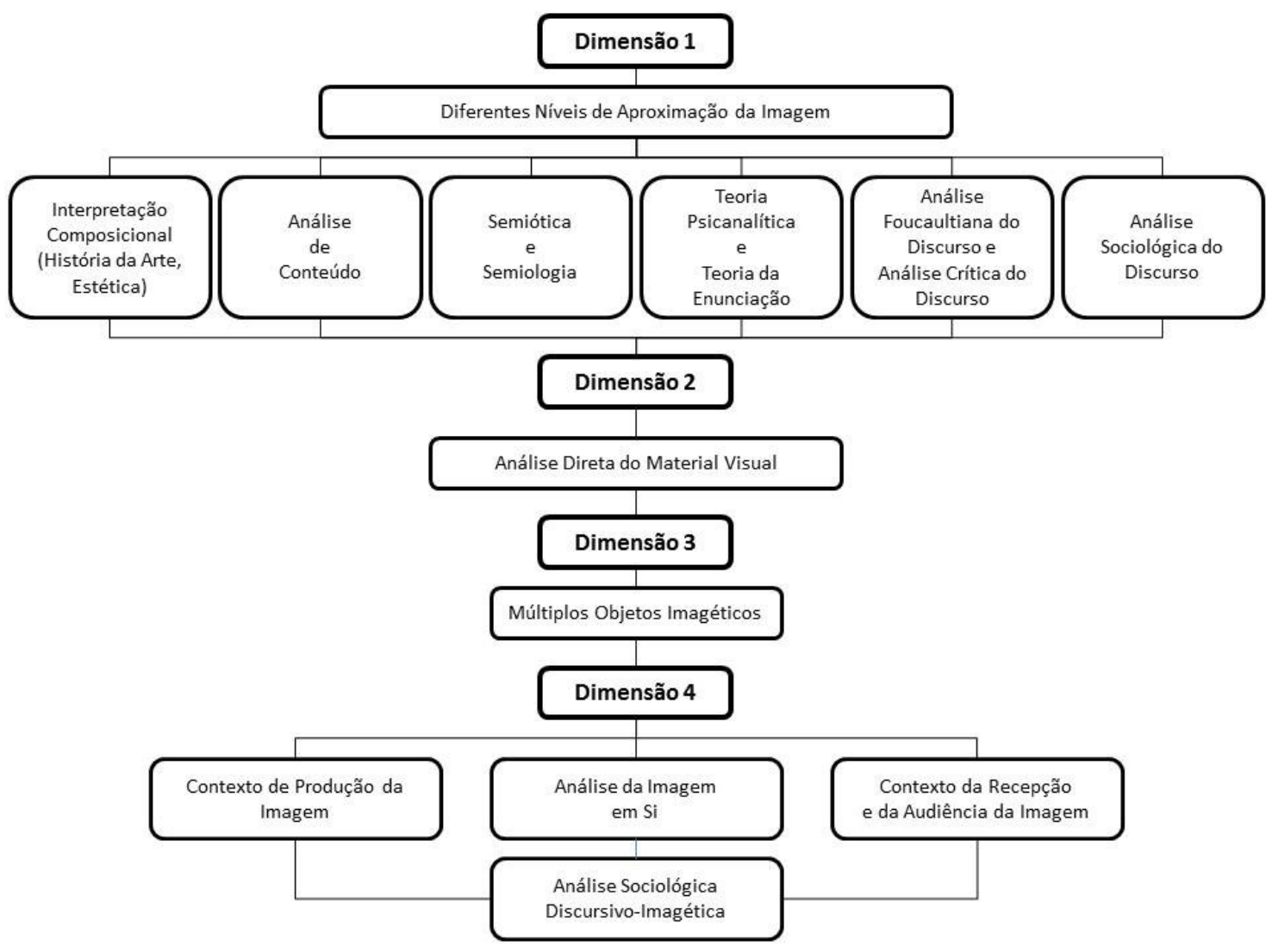

Figura 2 - Dimensões integradas e contextualizadas da Análise Sociológica Discursivo-Imagética

Fonte: Elaborado pelos autores.

\section{A INTERFACE DA ANÁLISE DE IMAGENS COM A ANÁLISE SOCIOLÓGICA DO DISCURSO: A PERSPECTIVA DA METODOLOGIA DA ANÁLISE SOCIOLÓGICA DA IMAGEM}

Vinculada à Tradição Espanhola de Investigação Social Qualitativa (IBÁÑEZ, 1986; ORTÍ, 2001; RUIZ RUIZ, 2009) emergiu, no início dos anos 1970, a chamada Análise Sociológica do Discurso (ASD) - atualmente já difundida no Brasil (ver, por exemplo: GODOI, 2009, 2010; GODOI; COELHO; SERRANO, 2014; COELHO; GODOI; COELHO, 2015). Conduzida pela fenomenologia, etnologia e teoria crítica da sociedade, a ASD consiste na busca de um modelo de representação e compreensão do texto concreto em seu contexto social e histórico, desde a reconstrução dos interesses conscientes e inconscientes dos atores envolvidos no discurso.

É nesse nível social-hermenêutico que o texto é concebido de forma abrangente e vincula-se diretamente com a dimensão mais pragmática da linguagem e a análise de seus usos sociais (CONDE, 2009). O que se pretende neste espaço de emergência e construção 
de uma metodologia de análise integrada de imagens e contextualizada no cenário social é estabelecer uma interface, realizada a partir do traslado e reconceituação dos procedimentos utilizados por Conde (2009). Tal aproximação justifica-se em virtude dos seguintes motivos: a) Rose (2012) aderiu à mesma sistemática na construção de sua metodologia visual-crítica, ou seja, utilizou-se de outras tendências textuais e discursivas; b) Serrano (2008) chegou a propor uma aproximação aparentemente metodologia discursivo-imagética, justapondo, sobre os estudos de Rose (2012) a ASD -, ocorre que até o momento, tal proposição não fora elaborada, tampouco sistematizada; c) a interface entre os procedimentos originários no interior da ASD e a metodologia de análise de imagens pretendida justifica-se também do ponto de vista epistemológico, uma vez que ambas constituem aqui epistemes sócio-hermenêuticas e pragmáticas, ou seja, contextualizadas por um sujeito social em um campo social. Infortunadamente, não é possível no espaço deste artigo estabelecer uma maior explanação sobre a história, a epistemologia, os conceitos e nem mesmo um maior detalhamento sobre os procedimentos da ASD, o que já fora anteriormente realizado em outros estudos no âmbito dos Estudos Organizacionais brasileiros.

Quadro 1 - Etapas e procedimentos da Metodologia de Análise Sociológica Discursivo-imagética

\begin{tabular}{|c|c|}
\hline \multicolumn{2}{|c|}{ I - Trabalhos Iniciais da Metodologia de Análise Sociológica Discursivo-Imagética } \\
\hline \multirow{3}{*}{$\begin{array}{l}\text { Anotações de campo } \\
\text { do pesquisador }\end{array}$} & $\begin{array}{l}\text { Os Procedimentos Iniciais consistem em tarefas conjuntas ou } \\
\text { imediatamente posteriores ao trabalho de campo: }\end{array}$ \\
\hline & $\begin{array}{l}\text { Anotações de insights, associações, possíveis modos de ver, } \\
\text { elementos importantes que se destacam prioritariamente na } \\
\text { análise do contexto da produção. }\end{array}$ \\
\hline & $\begin{array}{l}\text { Relato das primeiras intuições, sensações, ideias e conclusões } \\
\text { diante das imagens primárias ou secundárias. }\end{array}$ \\
\hline \multirow{6}{*}{$\begin{array}{l}\text { Preparação da análise } \\
\text { da imagem em si }\end{array}$} & $\begin{array}{l}\text { Identificação de temáticas significativas no conjunto de ima- } \\
\text { gens. }\end{array}$ \\
\hline & $\begin{array}{l}\text { Observação das imagens atentando para a mudança da forma } \\
\text { habitual de ver. }\end{array}$ \\
\hline & Estabelecimento da dinâmica de relações entre as imagens. \\
\hline & $\begin{array}{l}\text { Identificação de peculiaridades imagéticas geradoras de pistas } \\
\text { que possam conduzir a conjecturas pré-analíticas. }\end{array}$ \\
\hline & $\begin{array}{l}\text { Elaboração de um mapa de posicionamento das imagens entre } \\
\text { si em relação a objetivos do estudo. }\end{array}$ \\
\hline & $\begin{array}{l}\text { Análise conjunta do contexto social, histórico, político, ideo- } \\
\text { lógico, enfim, situacional, no qual a imagem foi produzida da } \\
\text { imagem. }\end{array}$ \\
\hline \multicolumn{2}{|c|}{$\begin{array}{l}\text { II - Procedimentos de Interpretação da Metodologia de Análise } \\
\text { Sociológica Discursivo-Imagética }\end{array}$} \\
\hline \multirow{3}{*}{$\begin{array}{l}\text { Conjectura } \\
\text { Pré-analíticas Discursi- } \\
\text { vo-Imagéticas }\end{array}$} & $\begin{array}{l}\text { Primeiras tentativas de construir um sentido inicial, indicativo e } \\
\text { geral para o conjunto das imagens em relação aos objetivos do } \\
\text { estudo. }\end{array}$ \\
\hline & $\begin{array}{l}\text { Análise, primeiramente aberta (atenção flutuante), atenta à } \\
\text { expressividade, indícios e evocações das imagens. }\end{array}$ \\
\hline & $\begin{array}{l}\text { Para a validação posterior, o conjunto das conjecturas deve ser } \\
\text { capaz de integrar, e explicar os objetivos da investigação. }\end{array}$ \\
\hline
\end{tabular}




\begin{tabular}{|c|c|}
\hline \multirow{2}{*}{$\begin{array}{l}\text { Estilos Discursivo-Ima- } \\
\text { géticos }\end{array}$} & $\begin{array}{l}\text { Representam uma forma de condensação de várias linhas } \\
\text { de condicionamentos inerentes ao contexto de produção da } \\
\text { imagem. }\end{array}$ \\
\hline & $\begin{array}{l}\text { Os condicionamentos que determinam os estilos imagéticos são } \\
\text { originários: da estrutura social e ideológica do contexto no qual } \\
\text { a imagem foi produzida; da sociedade em que a imagem está } \\
\text { inserida; da tecnologia de produção da imagem. }\end{array}$ \\
\hline \multicolumn{2}{|c|}{$\begin{array}{l}\text { III - Procedimentos de Análise da Metodologia de Análise Sociológica Discursivo-Imagé- } \\
\text { tica }\end{array}$} \\
\hline \multirow{5}{*}{$\begin{array}{l}\text { Posicionamento } \\
\text { Discursivo-Imagético }\end{array}$} & $\begin{array}{l}\text { Resposta aos questionamentos: Quem, Quando, Para Que, Por } \\
\text { que, Para quem? a imagem foi gerada. }\end{array}$ \\
\hline & Qual a intencionalidade da imagem? \\
\hline & $\begin{array}{l}\text { Perspectiva ou pontos de vista sobre o tema que as imagens } \\
\text { expressam. }\end{array}$ \\
\hline & $\begin{array}{l}\text { Análise dos diferentes efeitos posições visuais oferecidas pela } \\
\text { imagem em si. }\end{array}$ \\
\hline & $\begin{array}{l}\text { A ênfase recai sobre tanto sobre Contexto de Produção da } \\
\text { Imagem quanto sobre a Imagem Si }\end{array}$ \\
\hline \multirow{5}{*}{$\begin{array}{l}\text { Configurações } \\
\text { Narrativas Discursivo- } \\
\text {-Imagéticas }\end{array}$} & $\begin{array}{l}\text { Respostas aos questionamentos: Do que tratam a imagem? } \\
\text { Como se organizam os conteúdos das imagens entre si? }\end{array}$ \\
\hline & Relação das diferentes posições visuais entre si. \\
\hline & $\begin{array}{l}\text { Análise das tensões, conflitos diferenças entre posições imagéti- } \\
\text { cas e papéis desempenhados pelas imagens. }\end{array}$ \\
\hline & $\begin{array}{l}\text { Geração de uma primeira hipótese sobre dimensões, eixos ou } \\
\text { vetores de representação gráfica. }\end{array}$ \\
\hline & $\begin{array}{l}\text { A ênfase recai sobre tanto sobre o Contexto de Produção quanto } \\
\text { sobre a Análise da Imagem em Si (efeito visual). }\end{array}$ \\
\hline \multirow{6}{*}{$\begin{array}{l}\text { Espaços Semânticos } \\
\text { Discursivo-Imagéticos }\end{array}$} & $\begin{array}{l}\text { Respostas aos questionamentos: Qual a tecnologia de trans- } \\
\text { missão da imagem; O que a imagem transmite? De que forma } \\
\text { circula; Por quem é interpretada (audiência) }\end{array}$ \\
\hline & Análise do significado visual, das composições e efeitos visuais. \\
\hline & $\begin{array}{l}\text { Configuração e delimitação dos principais conteúdos e suas } \\
\text { materialidades visuais. }\end{array}$ \\
\hline & $\begin{array}{l}\text { Análise de como as imagens concretas vinculam-se ou disso- } \\
\text { ciam-se das do objeto de investigação. }\end{array}$ \\
\hline & $\begin{array}{l}\text { Relaciona-se com o "campo semântico" - conjunto de unidades } \\
\text { de significado, dotadas de organização estrutural subjacente } \\
\text { (contextos). }\end{array}$ \\
\hline & $\begin{array}{l}\text { A ênfase recai tanto sobre a Imagem em Si quanto sobre o } \\
\text { Contexto da Recepção da Imagem (audiência) }\end{array}$ \\
\hline $\begin{array}{l}\text { Relação entre Confi- } \\
\text { gurações Narrativas e } \\
\text { Espaços Semânticos }\end{array}$ & $\begin{array}{l}\text { Análise dos desajustes e distanciamento entre a análise das } \\
\text { configurações narrativas e dos espaços semânticos, em função } \\
\text { dos objetivos da pesquisa. }\end{array}$ \\
\hline \multicolumn{2}{|c|}{$\begin{array}{l}\text { IV - Procedimentos Complementares da Metodologia de Análise } \\
\text { Sociológica Discursivo-Imagética }\end{array}$} \\
\hline \multirow{3}{*}{$\begin{array}{l}\text { Análise das Conden- } \\
\text { sações }\end{array}$} & $\begin{array}{l}\text { Análise de temas, conteúdos, que possam parecer desconexos, } \\
\text { ilógicos, fora do lugar. }\end{array}$ \\
\hline & $\begin{array}{l}\text { Efeitos visuais que dão indícios de ligações psíquicas subjacen- } \\
\text { tes, enunciativas, latentes ou inconscientes. }\end{array}$ \\
\hline & $\begin{array}{l}\text { Análise de efeitos visuais metafóricos (uma coisa representando } \\
\text { outra) }\end{array}$ \\
\hline
\end{tabular}




\begin{tabular}{|l|l|}
\hline \multirow{4}{*}{$\begin{array}{l}\text { Análise dos Desloca- } \\
\text { mentos }\end{array}$} & $\begin{array}{l}\text { Análise de temas, conteúdos, expressões visuais, que assinalam } \\
\text { uma posição defensiva, conflito latente, repressão ou censura. }\end{array}$ \\
\cline { 2 - 2 } $\begin{array}{l}\text { Utilização de represen- } \\
\text { tações gráficas }\end{array}$ & $\begin{array}{l}\text { Análise de efeitos visuais metonímicos (deslocamentos em } \\
\text { cadeias associativas) }\end{array}$ \\
\hline & $\begin{array}{l}\text { Auxiliam na explicitação das conjecturas, isto é, permitem que } \\
\text { a intuição (ou conjectura implícita) na gênese do modelo possa } \\
\text { formalizar-se e evidenciar-se, distanciando-a desta mera forma } \\
\text { de intuição mais inefável. }\end{array}$ \\
\cline { 2 - 2 } & $\begin{array}{l}\text { Utilizam-se distintos formatos gráficos (topográficos): quadros, } \\
\text { matrizes, esquemas, figuras que ajudam a visualizar e ordenar } \\
\text { os resultados da análise realizada, bem como identificar dimen- } \\
\text { sões mais relevantes das linhas de análise e interpretação. }\end{array}$ \\
\hline
\end{tabular}

Fonte: Elaborado pelos autores.

Do ponto de vista epistemológico, em virtude da forma análoga com que fora construída, a ASDI guarda com a Análise Sociológica do Discurso (ASD), as mesmas influências epistemológicas, quais sejam: o marxismo; o estruturalismo francês; e a teoria psicanalítica. Além disso, assim como ASD, a ASDI constitui também uma abordagem metodológica sócio-hermenêutica e pragmática. Elaboramos, portanto, a partir da metodologia de ASD proposta por Conde (2009), sistematizada e sintetizada por Godoi, Coelho, e Serrano (2014), um quadro explicativo da metodologia aqui construída, ou seja, um conjunto de procedimentos norteadores dos modos de ver do pesquisador a partir dos quais pretendemos estabelecer as etapas de uma Metodologia de Análise Sociológica Discursivo-Imagética. Cabe observar que a expressão discursivo-imagética é originária não apenas do traslado proposto, mas da intenção de "ler imagens como discursos" (ROSE, 2012; SERRANO, 2008; UCHOA; GODOI, 2016; GODOI; UCHOA, 2016).

Cabem ainda algumas informações: a) A ASD (CONDE, 2009) prescreve a transcrição literal e direta do texto, o que por motivos já narrados não faz parte da metodologia aqui proposta; b) foram mantidas sob a forma de condensação, adaptação ou reconceituação a maior parte dos procedimentos de Interpretação e de Análise da ASD, e eliminados aqueles que exigiam atos da fala; c) o contexto de produção, a análise da imagem em si, e o contexto de recepção da imagem encontram-se imiscuídos entre os três Procedimentos de Análise da Metodologia Discursivo-Imagética proposta, encontrando, ênfase predominância sequencial na construção dos três Procedimentos de Análise.

Por fim, vale lembrar que os procedimentos da metodologia proposta não pretendem ser aplicados cegamente, de forma cronológica, demasiadamente sistemática, ou servir de como receituário, manual, protocolo a ser utilizado de forma independente do contexto particular. Trata-se de uma proposta metodológica "semiformal", na qual o pesquisador, ao exercitar, perceberá os encaixes diferenciados, a necessidade de criatividade e flexibilidade de tudo que é qualitativo, flexível e necessário de ser adaptado ao seu objeto de pesquisa.

\section{CONSIDERAÇÕES FINAIS}

O desafio do estudo das imagens é assumido como uma tarefa que incita a repensar a cultura visual-material ou, simplesmente, o modo visual (MEYER et al., 2013) 
pelo qual se manifestam os artefatos e discursos na contemporaneidade - lócus onde as imagens ocupam, à revelia da ortodoxia da pesquisa social discursiva, centralidade. Aqui não se trata mais de separar, tricotomizar texto-discurso-imagem (por certo que texto e imagem suscitam diferentes efeitos "emocionais", diferença esta essencialmente atribuída ao imediatismo da imagem). Há que se considerar, no entanto, que o modo visual de vida refere-se a uma forma particular de construção e expressão do significado, forma esta que não temos mais como fazer retroceder: o significado é criado, transformado transferido e colocado em prática por meio do uso prioritário de objetos e artefatos ou, no dizer de Meyer et al. (2013), pela integração do visual e do verbal numa relação na qual uma esfera não pode mais ser compreendida sem a outra.

Acerca da ética na pesquisa com imagens, Lapenta (2011) alerta que a inclusão de imagens de outras pessoas passíveis de identificação pode gerar problemas éticos, bem como fotografias que não representem o contexto original, gerando interpretações errôneas. Howell et al. (2014) e Wiles, Clark e Prosser (2011) acrescentam que a grande flexibilidade dos diversos métodos de pesquisa visual e sua interdisciplinaridade (PINK, 2003) exigem uma forte negociação sobre o terreno da ética. No entender de Pink (2013) os problemas éticos relacionados à etnografia vão além de uma simples conduta ética do pesquisador, mas atingem à compreensão ética sobre o contexto de pesquisa, ou seja, trata-se de uma abordagem reflexiva do pesquisador sobre suas próprias crenças e uma abordagem crítica sobre um código de conduta ética hierarquicamente superior aos demais. Uma complexidade, que segundo Banks (2012), expõe a origem de seus entrevistados, o que de fato fazem e não o que dizem fazer.

Na compreensão de Banks (2012) a preocupação com a questão ética nas práticas visuais é necessária a fim de evitar a exposição das origens de seus entrevistados. Os vídeos, em pesquisa participante, têm sido utilizados de várias maneiras em ciências sociais buscando minimizar esse dilema. Os participantes são encorajados a construir seus próprios textos e vídeos em uma perspectiva de envolvimento sob a égide de vídeo colaborativo (PINK, 2013), vídeo comunidade (MITCHELL; LANGE, 2012), e vídeo participativo (MISTRY et al., 2014) - processos que caracterizam um mínimo de colaboração do pesquisador e sua equipe.

Independente do veículo analítico, estamos em Estudos Organizacionais limitados, até o momento, a entender que o material visual somente permite coletar situações, não conceitos. Por outro lado, podemos admitir que diários de campo (por exemplo, fieldnotes com texto e imagem - prática ainda inovadora) podem constituir fortes aliados na construção do contexto, na delimitação do objeto, na ausência de um evento-objeto organizacional previamente construído. Uma das principais contribuições da utilização de recursos visuais na prática da pesquisa é a imprescindibilidade que gera ao pesquisador de sair do laboratório e investigar acerca do campo, construí-lo, pois é justamente ali que reside a cotidianeidade significativa capaz de encarnar a prática transformadora da investigação.

A Metodologia da Análise Sociológica Discursivo-Imagética aqui construída pretende trazer como contribuição central o preenchimento da lacuna acerca da inexistência, até hoje, de uma metodologia capaz de analisar diretamente imagens sem antes fazer uma transcrição textual e, posteriormente, aplicar uma técnica textual ou discursiva pré-existente, perdendo assim todos os elementos não verbais da imagem, como emocionalidades, por 
exemplo. Além deste problema em relação à imagem em si, o que se perde em todas as metodologias analisadas, mesmo naquelas não uni-objetais são os elementos contextuais da imagem (contexto histórico, social, ideológico, político, econômico) e os elementos contextuais (ligados a qual audiência se destina; tipo de tecnologia e veículos utilizados na geração e transmissão da imagem). Tais deficiências foram o que procuramos solucionar com a construção de uma proposta integradora e contextualizada.

\section{AGRADECIMENTOS}

Agradecemos à Coordenação de Aperfeiçoamento de Pessoal de Nível Superior (CAPES) pela concessão da Bolsa de Estágio Doutoral (BEX 6450/15-6), a qual nos possibilitou desenvolver a presente pesquisa.

\section{REFERÊNCIAS}

ALCADIPANI, R.; TONELLI, M. Violence, masculinity and the shop floor. Gender, Work Organization, v. 4, n. 21, p. 321-340, 2014.

ALPERS, S.; VERMEER, J. The art of describing: Dutch art in the seventeenth century. Chicago: University of Chicago Press, 1983.

ARROYO, M. Los análisis de imagen y de percepciones sociales. In: ARROYO MENÉNDEZ, M. A.; SÁBADA RODRÍGUEZ, I. Metodología de la investigación social: técnicas innovadoras y sus aplicaciones. Madrid: Editorial Sínteses, 2012. cap. 15.

ARROYO, M.; SÁBADA, I. Metodología de la investigación social: técnicas innovadoras y sus aplicaciones. Madrid: Editorial Sínteses, 2012.

BANKS, M. The Place of Visual Data in Social Research: A Brief History. In: HUGHES, J. SAGE Visual Methods. London: Sage, 2012.

BANKS, M.; ZEITLYN, D. Visual methods in social research. 2. ed. London: Sage, 2015.

BATESON, G.; MEAD, M. Balinese character: A photographic analysis. In: ROBBEN, A. C. G. M.; SLUKA, J. A. Ethnographic Fieldwork: An Anthropological Reader. 2. ed. Wiley-Blackwell, 2012.

CALDAS, M.; TONELLI, M. J. Casamento, estupro ou dormindo com o inimigo: interpretando imagens e representações dos sobreviventes de fusões e aquisições. Organizações \& Sociedade, v. 9, n. 23, p. 171-186, 2002.

CHALFEN, R. Looking Two Ways: Mapping the Social Scientifc Study of Virtual Culture. In: MARGOLIS, E.; PAUWELS, L. The SAGE Handbook of Visual Research Methods. London: Sage, 2011.

COELHO, A. L. D. A. L.; GODOI, C. K.; COELHO, C. Análise Sócio-hermenêutica do Discurso da Sustentabilidade a Partir de Materiais Visuais. RAC - Revista de Administração Contemporânea, v. 19, n. 5, p. 649-670, 2015. 
COLLIER JR, J. Photography in Anthropology: A Report on Two Experiments. American Anthropologist, v. 59, n. 5, p. 843-859, 1957.

COLLIER, J.; COLLIER, M. Visual anthropology: Photography as a research method. Abuquerque: University of New Mexico Press, 1986.

CONDE, F. Análisis sociológico del sistema de discursos. Cuadernos Metodológicos, n. 43. Madrid: Centro de Investigaciones Sociológicas (CIS), 2009.

CUNLIFF, A. L.; ALCADIPANI, R. The politics of access in fieldwork: Immersion, backstage dramas, and deception. Organizational Research Methods, v. 19, n. 4, p. 535-561, 2016.

FELSTEAD, A.; JEWSON, N.; WALTERS, S. Images, interviews and interpretation: making connections in visual research. Studies in Qualitative Methodology, Leicester, n. 7, 2004. p. 105-121.

FLICK, U. Introducción a la investigación cualitativa. Madrid: Morata, 2004.

GARCÍA-VERA, A. B.; MAILLO, H. M. V. Antropología audiovisual: medios e investigación en educación. Madrid: Ed. Trotta, 2011.

GARFINKEL, H.; LYNCH, M.; LIVINGSTON, E. The work of a discovering science constructed with materials from the optically discovered pulsar. Philosophy of the social sciences, $v$. 11 , n. 2, p. 131-158, 1981.

GODOI, C. K. Perspectivas de análise do discurso nos estudos organizacionais. In: GODOI, C. K.; BANDEIRA DE MELLO, R.; SILVA, A. B. Pesquisa qualitativa em estudos organizacionais: paradigmas, estratégias e técnicas. São Paulo: Saraiva, 2010. p. 375-401.

A perspectiva da interpretação social dos discursos: uma prática de análise dos discursos motivacionais na aprendizagem com base nos atos da fala, enunciação e contexto. In: CARRIERI, A. P. Análise do discurso em estudos organizacionais. Curitiba: Juruá, 2009. p. $131-152$.

GODOI, C. K.; COELHO, A. L. D. A. L.; SERRANO, A. Elementos epistemológicos e metodológicos da análise sociológica do discurso: abrindo possibilidades para os estudos organizacionais. Organizações \& Sociedade, v. 21, n. 70, p. 509-535, 2014.

GODOI, C. K.; UCHOA, A. G. F. Metodologia Qualitativa Discursivo-Imagética: do contexto da produção às possibilidades de recepção da imagem. In: CONGRESSO BRASILEIRO DE ESTUDOS ORGANIZACIONAIS. 4., 2016, Porto Alegre. Anais .... Porto Alegre: UFRGS, 2016.

GUASCH, A. Los estudios visuales. Un estudio de la cuestión. Arte e Investigación. La Plata, v. 10, n. 5, p. 10-18, 2006.

HARPER, D. Reimagining visual methods: Galileo to neuromancer. In: DENZIN, N. K.; LINCOLN, Y. S. (eds.). Collecting and interpreting qualitative materials. Thousand Oaks, CA: Sage, 2003. p. 176-198.

. An argument for visual sociology. In: PROSSE, J. Image-based research: A sourcebook for qualitative researchers. London: Routledge, 2000, p. 24-41.

HOWELL, C. et al. Exploring ethical frontiers of visual methods. Research Ethics, v. 10, n. 4, p. 208-213, 2014. 
HUGHES, J. SAGE Visual Methods. London: Sage, 2012.

IBÁÑEZ, J. Más allá de la sociología. El grupo de discusión: teoría y crítica. 5. ed. Madrid: Sieglo Veintiuno Editores, 2003.

IBÁÑEZ, J. Perspectivas de la investigación: el diseño en las tres perspectivas. In: GARCÍA, M.; IBÁÑEZ, J.; ALVIRA, F. El análisis del realidad social: métodos y técnicas de investigación. Madrid: Alianza, 1986.

JACKSON, S. Performing show and tell: disciplines of visual culture and performance studies. Journal of Visual Culture, v. 4, n. 2, p. 163-177, 2005.

JACKSON, B. Killing Time: Life in the Arkansas Penitentiar. Ithaca, NY: Cornell University, 1977.

LAPENTA, F. Some Theoretical and Methodological Veiws on Photo-Elicitation. In: MARGOLIS, E.; PAUWELS, L. The SAGE handbook of Visual Research Methods. London: Sage, 2011.

LIEBENBERG, L. The visual image as discussion point: increasing validity in boundary crossing research. Qualitative Research, v. 9, n. 4, 2009, p. 441-467.

MARGOLIS, E.; ROWE, J. Methological Approaches to Disclosing Historic Photographs. In: MARGOLIS, E.; PAUWELS, L. The SAGE Handbook of Visual Research Methods. London: Sage, 2011.

MEAD, M. Visual Anthropology in a Discipline of Words. In: HOCKINGS, P. Principles of Visual Anthropologies. 3. ed. Berlin: Walter de Gruyter GmbH \& Co. KG, 2003.

MENÉNDEZ, M. A. A.; RODRÍGUEZ, I. S. Metodología de la investigación social: técnicas innovadoras y sus aplicaciones. Madrid: Editorial Sínteses, 2012.

MENESES, U. T. B. Fontes visuais, cultura visual, História visual. Balanço provisório, propostas cautelares. Revista Brasileira de História, São Paulo, v. 23, n. 45, p. 11-36, 2003.

MEYER, R. E. et al. The Visual Dimension in Organizing, Organization, and Organization Research: Core Ideas, Current Developments, and Promising Avenues. The Academy of Management Annals, v. 7, n. 1, p. 489-555, 2013.

MIRZOEFF, N. Una introducción a la cultura visual. Barcelona: Paidós, 2003. p. 239-254.

. Ghostwriting: working out visual culture. Journal of Visual Culture, v. 1, n. 2, 2002,

MISTRY, J. et al. The role of social memory in natural resource management: insights from participatory video. Transactions of the Institute of British Geographers, Plymouth, v. 39, n. 1, p. 115-127, 2014.

MITCHELL, C.; LANGE, N. D. Handbook of participatory video. Plymouth: AltaMira Press, 2012.

. Community-Based Participatory Video and Social Action in Rural South Africa. In: MARGOLI, E.; PAWELS, L. The SAGE Handbook of Visual Research Methods. London: Sage, 2011. 
MONDADA, L. Using video for a sequential and multimodal analysis of social interaction: Videotaping institutional telephone calls. Forum Qualitative Sozialforschung/Forum: Qualitative Social Research. 2008.

ORTí, A. En el margen del centro: la formación de la perspectiva sociológica crítica de la generalización de 1956. Revista Española de Sociologia, v. 1, p. 119-163, 2001.

O'TOOLE, P.; WERE, P. Observing places: unsing space and material culture in qualitative research. Qualitative Research, v. 8, n. 5, 2008, p. 616-634.

PAUWELS, L. An Integrated Conceptual Framework for Visual Social Research. In: MARGOLIS, E.; PAUWELS, L. The SAGE Handbook of Visual Research Methods. London: Sage, 2011.

PINK, S. Doing visual ethnography. London: Sage, 2013.

. Interdisciplinary agendas in visual research: re-situating visual anthropology. Visual studies, v. 18, n. 2, p. 179-192, 2003.

REBOLLO, J. G. Antropologia visual: fundamentos teóricos y metodológicos en inserción del audiovisual en diseños de investigación social. Barcelona: Bellaterra, 2002.

RIEGER, J. A retrospective visual study of social change: the pulp-logging industry in an Upper Peninsula Michigan county. Visual Studies, v. 18, n. 2, p. 157-178, 2003.

ROSE, G. Visual methodolgies: an introduction to resourcing with visual materials. London: Sage, 2012.

RUIZ RUIZ, J. Análisis sociológico del discurso: métodos y lógicas. Forum Qualitative Sozialforschung, v. 10, n. 2, 2009.

SCHWARTZ, D. Visual ethnography: Using photography in qualitative research. Qualitative sociology, v. 12, n. 2, p. 119-154, 1989.

SERRANO, A. El análisis de materiales visuales en la investigación social: el caso de la publicidad. In: GORDO, A.; SERRANO, A. Estrategias y práticas cualitativas de investigación social. Madrid: Pearson Prentice Hall, 2008.

SERRANO, A. P. L.; ZURDO, Á. A. Investigación social con materiales visuales. In: ARROYO MENÉNDEZ, M. A.; SÁBADA RODRIGUEZ, I. Metodología de la investigación social: técnicas innovadoras y sus aplicaciones. Madrid: Editorial Sínteses, 2012. Cap. 10.

SHAW, J. Interrogating the GAP between the Ideals and Pratice Reality of Paticipatory Video. In: MILNE, E.-J.; MITCHELL, C.; LANGE, N. D. Handbook of Participatory Video. Plymouth: Altamira Press, 2012. p. 225-241.

THOMAS, N. Entangled objects: exchange, material culture, and colonialism in the Pacific. Cambridge: Harvard University Press, 2009.

UCHOA, A. G. F.; GODOI, C. K. Metodologias Qualitativas de Análise de Imagens: origem, historicidade, diferentes abordagens e técnicas. In: CONGRESSO BRASILEIRO DE ESTUDOS ORGANIZACIONAIS. 4., 2016, Porto Alegre. Anais .... Porto Alegre: UFRGS, 2016.

WAGNER, J. Seeing Things: Visual Research and Material Culture. In: MARGOLIS, E.; PAUWELS, L. The SAGE Handbook of Visual Research Methods. London: Sage, 2011a. 
WAGNER, J. Visual Studies and Empirical Social Inquiry. In: MARGOLIS, E.; PAUWELS, L. The SAGE Handbook of Visual Research Methods. London: Sage, 2011b.

WILES, R.; CLARK, A.; PROSSER, J. Visual Research Ethics at the Crossroads. In: MARGOLIS, E.; PAUWELS, L. The SAGE Handbook of Visual Research Methods. London: Sage, 2011.

Data de Submissão: 24/02/2018.

Data de Aprovação: 25/09/2018. 